\title{
Neutronics and activation of the preliminary reaction chamber of HiPER reactor based in a SCLL blanket
}

\author{
Rafael Juárez , Javier Sanz , A.J. Lopez-Revelles , José Manuel Perlado
}

\section{H I G H L I G H T S}

- Neutronic study of a proposal of a reaction chamber for HiPER reactor.

- Two options for the blanket size, thin and thick, are studied and compared.

- The thin blanket performs better than the thick blanket.

- The proposed Vacuum Vessel is unviable as lifetime component in both cases.

- Likely solutions for the Vacuum Vessel lifetime extension are explored.

\begin{abstract}
A B S T R A C T
The HiPER reactor design is exploring different reaction chambers. In this study, we tackle the neutronics and activation studies of a preliminary reaction chamber based in the following technologies: unprotected dry wall for the First Wall, self-cooled lead lithium blanket, and independent low activation steel Vacuum Vessel. The most critical free parameter in this stage is the blanket thickness, as a function of the ${ }^{6} \mathrm{Li}$ enrichment. After a parametric study, we select for study both a "thin" and "thick" blanket, with "high" and "low" ${ }^{6} \mathrm{Li}$ enrichment respectively, to reach a TBR=1.1. To help to make a choice, we compute, for both blanket options, in addition to the TBR, the energy amplification factor, the tritium partial pressure, the ${ }^{203} \mathrm{Hg}$ and ${ }^{210} \mathrm{Po}$ total activity in the LiPb loop, and the Vacuum Vessel thickness required to guarantee the reweldability during its lifetime. The thin blanket shows a superior performance in the safety related issues and structural viability, but it operates at higher ${ }^{6} \mathrm{Li}$ enrichment. It is selected for further improvements. The Vacuum Vessel shows to be unviable in both cases, with the thickness varying between 39 and $52 \mathrm{~cm}$. Further chamber modifications, such as the introduction of a neutron reflector, are required to exploit the benefits of the thin blanket with a reasonable Vacuum Vessel.
\end{abstract}

\section{Introduction}

The HiPER project contemplates the design of an IFE reactor based in the reasonable extrapolation of the technologies developed during the HiPER engineering phase [1]. Thus, the HiPER reactor will operate at $10 \mathrm{~Hz}$ with $150 \mathrm{MJ}$ fusion yields, making use of direct drive ignition. It will be based in unprotected dry wall technologies for the First Wall (FW). In this framework, the HiPER project contemplates the study of a preliminary reaction chamber based in a self-cooled lead lithium (SCLL) blanket with an independent low activation steel Vacuum Vessel (VV), as will be described in the next section. In this paper, we address the neutronic and activation performance of such a reaction chamber for two different blanket thickness options.

There are multiple combinations of breeder thickness and ${ }^{6} \mathrm{Li}$ enrichment in the $15.8 \mathrm{Li}$ at.\% of eutectic $\mathrm{LiPb}$ which guarantee a $\mathrm{TBR}=1.1$, which is a design objective for HiPER reactor. We select for study in this paper the two trends: thin blanket with high ${ }^{6} \mathrm{Li}$ enrichment and thick blanket with low ${ }^{6} \mathrm{Li}$ enrichment. For these blanket options, we compute the energy amplification factor and the TBR range as a function of the ${ }^{6} \mathrm{Li}$ enrichment, for flexible operation, remarked as mandatory in [2].

The blanket thickness has impact not only on in the ${ }^{6} \mathrm{Li}$ enrichment and blanket performance, but also in the radiological safety and the Vacuum Vessel viability, among others. To support a design choice of the blanket thickness, we carry out in this paper a neutronic and activation study of the two blanket options.

Related to the radiological safety, we compute, for both LiPb loops, the total inventories of tritium, ${ }^{210} \mathrm{Po}$ and ${ }^{203} \mathrm{Hg}$, the main 


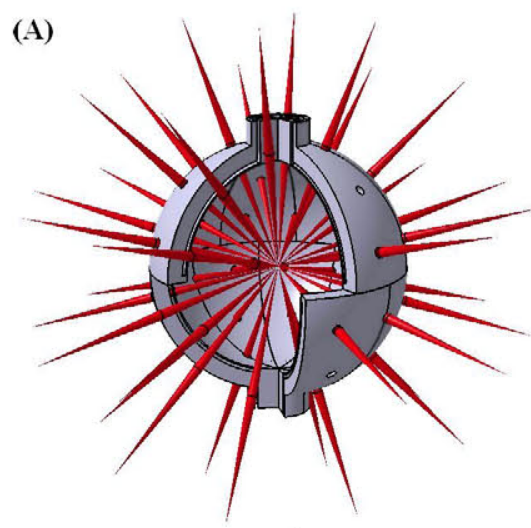

(B)
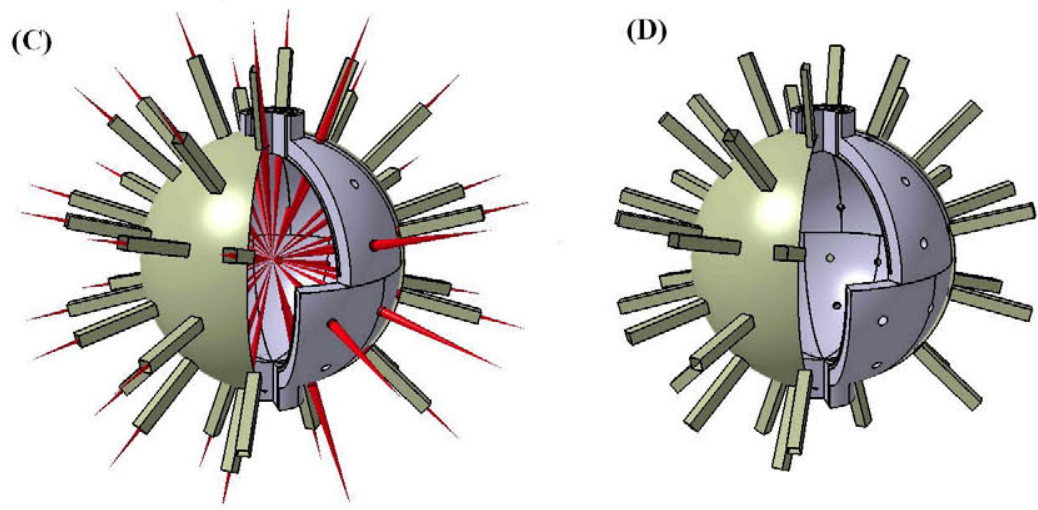

(E)

(F)
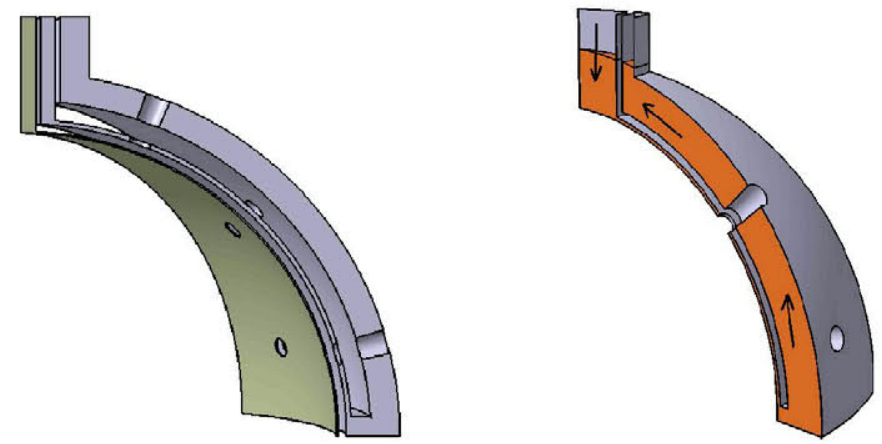

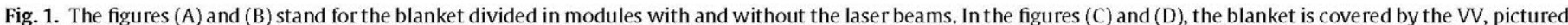

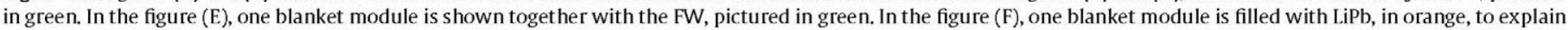
the LiPb circuit inside the blanket. (For interpretation of the references to color in the text, the reader is referred to the web version of the article.)

radiological concerns derived from the use of $\mathrm{LiPb}$ as tritium breeder [3]. The design guideline for this preliminary HiPER reaction chamber is taken from [4], and states that HiPER reactor design should avoid the need for an external evacuation plan in case of an accidental situation.

Related to the Vacuum Vessel design, we compute for both blanket options the dpa and the He transmutation rate, in order to assess the viability of the VV as a lifetime component of the facility. Some solutions are addressed in case that the radiation damage to the $\mathrm{VV}$ should be reduced.

\section{Preliminary reaction chamber for HiPER reactor}

The reaction chamber under study in this paper is a preliminary proposal accepted for study for the HiPER reactor. It is decoupled into three physically independent components, as shown in the Fig. 1: First Wall, Tritium Breeding Blanket and Vacuum Vessel.

The decoupling approach has been followed in recent dry wall IFE reaction chamber designs, such as LIFE [5], HAPL [6]. It presents multiple advantages, such as specialization of components and independent maintenance schemes.

The FW is placed at $R=6.5 \mathrm{~m}$ from the ignition point. It is considered to be composed of $1 \mathrm{~mm}$ of tungsten mounted onto $1 \mathrm{~cm}$ of EUROFER97. The survivability of this component has been addressed in other studies inside the HiPER project [7], and it is out of the scope of this work. The FW cooling is still under discussion in the HiPER project [8], but for this study it is assumed that the FW heat removal will be through the blanket cooling circuit.

The tritium breeding blanket starts after the FW. It presents a spherical shell shape, and it is divided into 16 modules, following the laser beams symmetries [1]. Thus, it is divided into two hemispheres, each one divided into eight parts. Each one of the 16 modules presents two channels through which the LiPb flows. The entrance channel is in direct contact with the FW and it is relatively thin $(8 \mathrm{~cm})$. These two decisions seek to favor the heat removal from the FW and the initial neutron power deposition in the LiPb by means of a fast mass flow. It takes advantage of the lower corrosion of the "cold" $\mathrm{LiPb}\left(350^{\circ} \mathrm{C}\right)$. Once the LiPb reaches the equator of 
Table 1

Summary of the neutronic and activation results for both thin and thick blanket. The accumulative quantities are shown after 40 years of irradiation.

\begin{tabular}{lll}
\hline & Thin blanket & Thick blanket \\
\hline TBR & 1.1 & 1.1 \\
Inner ch. thickness & $8 \mathrm{~cm}$ & $8 \mathrm{~cm}$ \\
Outer ch. thickness & $42 \mathrm{~cm}$ & $67 \mathrm{~cm}$ \\
LiPb volume & $310 \mathrm{~m}^{3}$ & $475 \mathrm{~m}^{3}$ \\
6Li enrich & $70 \%$ & $25 \%$ \\
$\eta$ blanket & 1.04 & 1.09 \\
TBR range & $0.66-1.21$ & $0.89-1.36$ \\
T p. pressure & $3 \mathrm{mPa}$ & $3 \mathrm{mPa}$ \\
T inventory $@$ 40 years & $61 \mathrm{mg}$ & $93 \mathrm{mg}$ \\
210 Po DRC @ 40 years & 4140 & 18,110 \\
${ }^{203}$ Hg DRC @ 40 years & 11.6 & 12.3 \\
VV dpa 94 years & 50 & 17 \\
VV thickness & $52 \mathrm{~cm}$ & $39 \mathrm{~cm}$ \\
\hline
\end{tabular}

the blanket, it turns around and exits the blanket at $480^{\circ} \mathrm{C}$ through a thick channel, which dimensions are to be determined in this paper with neutronic criteria (see Table 1 ). In the thick channel the mass flow is slower, given that the hot LiPb produces a higher corrosion rate.

All the heat deposited in the blanket (and also in the FW) is removed by the LiPb, and thus, it is a self-cooled lead lithium blanket. However, differently to the previous MFE SCLL blankets built of SiC, such as PPCS-D [9], the HiPER SCLL structure is fully built of $1 \mathrm{~cm}$ thick EUROFER97 sheets, what significantly relaxed the technological risk. This is possible given the absence of magnetic fields in the HiPER reactor. Further studies will hinder into the correct sheets thickness with structural criteria. The corrosion rate will be a threat to the reactor which is under consideration in other studies [8].

The VV starts at $R=8 \mathrm{~m}$, which is the maximum allowable distance before the final lenses, and it is assumed to be spherical in this preliminary proposal. As the VV has been decided to be a lifetime component, its thickness will be determined to guarantee the reweldability of the external face of the VV at 40 years.

\section{Neutronics and activation}

To support the reaction chamber design evolution, we compute some neutronic and activation quantities divided into three groups: operational, radiological safety related, and VV related quantities. The results are shown in Table 1. The computational methodology is explained in Section 3.1 and the irradiation scheme is detailed in Section 3.2

The operational quantities are: TBR, TBR margins, and energy amplification factor, $\eta_{\text {blanket }}$. The first and most important one is the TBR. It has been decided to design the HiPER reaction chamber with a TBR=1.1. As shown in Fig. 2, there are several combinations of breeder thickness and ${ }^{6} \mathrm{Li}$ enrichment leading to this TBR. We select for study the two extreme trends, thin blanket with high enrichment ( $50 \mathrm{~cm}, 70$ at.\% enrichment) and thick blanket with low enrichment $(75 \mathrm{~cm}, 25$ at.\% enrichment), for a deeper study and comparison.

The TBR margins account for the TBR variation depending on the ${ }^{6} \mathrm{Li}$ enrichment of the $\mathrm{LiPb}$, from 10 at.\% to 90 at.\%. It is a relevant quantity for the TBR online adjustment, what has been identified as critical [2]. The wider it is, the more flexible operation the blanket allows. The other operational quantity is the energy amplification factor, $\eta_{\text {blanket }}$, defined as the available energy in the blanket from neutron energy deposition divided by the energy carried by the neutrons. It has to be as high as possible.

The radiological safety related quantities considered in this paper are linked to the most worrying isotopes derived from the use of LiPb [3]: tritium, ${ }^{210} \mathrm{Po}$ and ${ }^{203} \mathrm{Hg}$. The tritium total inventory

\section{TBR vs. Breeder thickness}

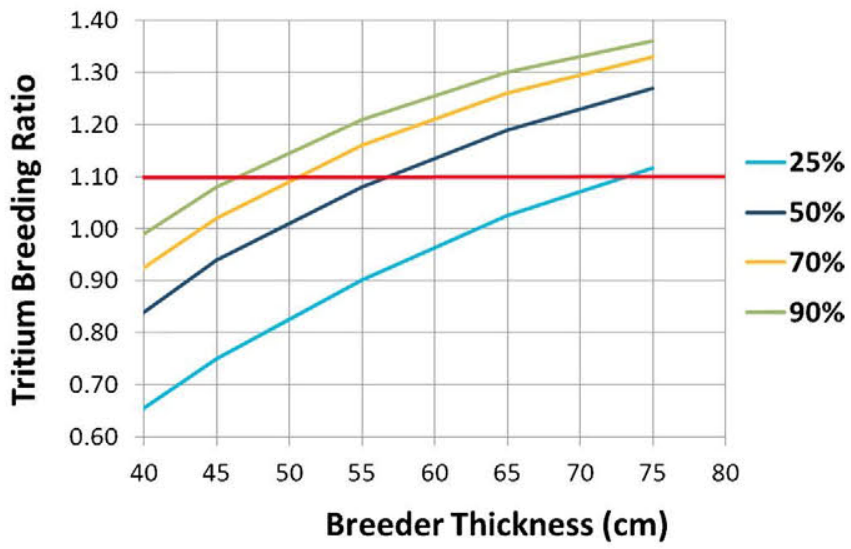

Fig. 2. Tritium breeding ratio as a function of the breeder thickness for different ${ }^{6} \mathrm{Li}$ enrichment of the LiPb.

in the LiPb loop indicates the amount of $T$ mobilizable in case of accident from the LiPb. Although it is anticipated that it will not probably be relevant compared with the T inventory in other reactor components [3], it allows a comparison between the thin and the thick blanket. It has been assumed a tritium extraction system with an efficiency of $90 \%$, and that the whole LiPb passes through the permeator in each cycle. The ${ }^{203} \mathrm{Hg}$ and the ${ }^{210}$ Po are dangerous isotopes of concern to be considered when seeking the no need for an external evacuation plan in case of accident. The release limits for these isotopes, $0.92 \mathrm{TBq}$ for ${ }^{210} \mathrm{Po}$ and $925 \mathrm{TBq}$ for ${ }^{203} \mathrm{Hg}$, are taken from [3]. As HiPER project has not yet decided a placement for the facility, those have been taken for being part of a representative study in the subject. We compute the degree of radiological confinement, DRC, as the division of the total isotope inventory in the loop (4 times the blanket volume) and the limit inventory to be released in case of accident. The design of safety systems will have to assure the DRC in the worst accidental situation. All the safety related quantites are shown after 40 years of irradiation.

The VV related quantities are the accumulated dpa and the accumulated He transmuted, both at 40 years, the facility expected lifetime. The accumulated dpa must be below 100 to guarantee the VV structural integrity. And the VV thickness should be adjusted to guarantee an accumulated transmuted He below 1 appm at 40 years in the external surface of the vessel. This last condition is mandatory to allow the reweldability. A similar approach was followed by HAPL team [6].

\subsection{Computational methodology}

The computational methodology used in this work is composed by four codes: CATIA V5R19, MCAM [10], MCNPX [11] and $A C A B$ [12]. They have been used respectively for the following task: 3D geometry design, translation from CATIA files to MCNPX available input, radiation transport and radiological inventory. The transport calculations have been performed using the JEFF-3.1.1 cross-sections library [13], the inventory calculations with the EAF2010 cross-sections library [14], and a processed library based in the ENDF/B-VII library [15] and the NRT model.

The materials compositions used in the calculations correspond to those found in EUROFER97 [16], LiPb [3]. It is to remark that the Bi concentration in the LiPb is $43 \mathrm{wppm}$. The neutron spectrum used corresponds to a re-escaling of the $157 \mathrm{MJ}$ yield of direct drive shock ignition computed in [17]. 


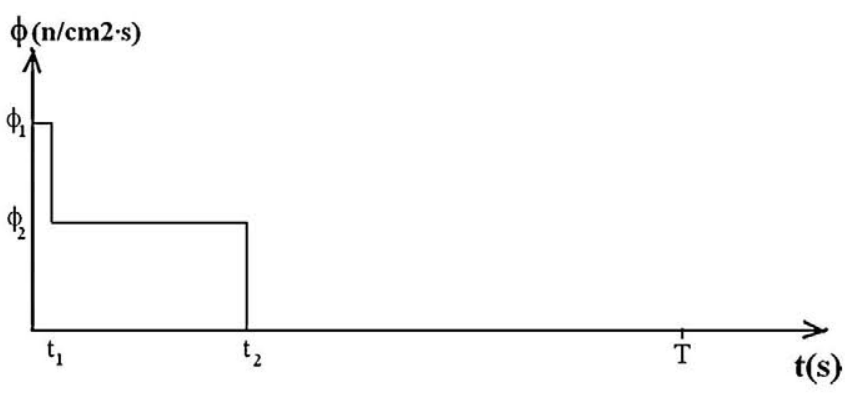

Fig. 3. Structure of one cycle of irradiation of the LiPb.

\subsection{Irradiation scheme}

The LiPb mass flow is $6.34 \mathrm{~m}^{3} / \mathrm{s}$. The LiPb, as mentioned, enters the blanket through an inner channel and exits through an outer channel, and the time it spends is the residence time; after that, it spends a three times longer period outside the blanket before re-entering. The residence time, under irradiation, has been modeled, thus, as two constant neutron fluxes $\phi_{1}$ from 0 to $t_{1}$, and $\phi_{2}$ from $t_{1}$ to $t_{2}$, one for each channel. The residence time, plus the additional period outside the blanket, is the cycle to be repeated in time, lasting $T$ seconds. The blanket options differ in the outer channel thickness, being the inner channel thickness common to both as indicated in the Table 1 .

In the Fig. 3 it is shown the irradiation structure explained before. The values of the different parameters for the two blanket options are shown in the Table 2.

\section{Vacuum Vessel as lifetime component}

As shown in the Table 1, the thick blanket offers better shielding to the VV than the thin blanket. However, both options are judged to be unsuitable, as steel sheet thicknesses larger than $20 \mathrm{~cm}$ are required. Consequently, a different strategy to the increase of the VV thickness is required to assure the reweldability after 40 years of irradiation with the blanket proposal under study.

Two approaches are explored in this section for the case of the thin blanket: the increase in the distance from the ignition to reduce the neutron flux in the VV, and the introduction of a graphite neutron reflector between the blanket and the $\mathrm{VV}$ as neutron shielding.

The increase of the distance is motivated, not only for VV maintenance, but also because of foreseen blanket and FW maintenance and structural support troubles given the reduced gap between the blanket and the VV (in the range of $50-75 \mathrm{~cm}$ ). This option required the modification of the distance from optics to the ignition point. The introduction of the graphite neutron reflector is motivated for two reasons, in addition to the protection of the VV: it is a light material, necessary given the weight of the blanket; it may increase the TBR, allowing a reduction of the ${ }^{6} \mathrm{Li}$ enrichment and reducing the operation costs. In the Fig. 4 it is shown the VV thickness performance using both approaches combined and independently for the thin blanket reaction chamber.

Table 2

Irradiation parameters of the thin and the thick blanket.

\begin{tabular}{lcc}
\hline & Thin blanket & Thick blanket \\
\hline$\phi_{1}\left(n / \mathrm{cm}^{2} \mathrm{~s}\right)$ & $1.61 \times 10^{15}$ & $2.34 \times 10^{15}$ \\
$\phi_{2}\left(n / \mathrm{cm}^{2} \mathrm{~s}\right)$ & $6.13 \times 10^{14}$ & $8.66 \times 10^{14}$ \\
$t_{1}(\mathrm{~s})$ & 7 & 7 \\
$t_{2}(\mathrm{~s})$ & 49 & 68 \\
$T(\mathrm{~s})$ & 196 & 300 \\
n. cycles/year & 160,898 & 105,120 \\
\hline
\end{tabular}

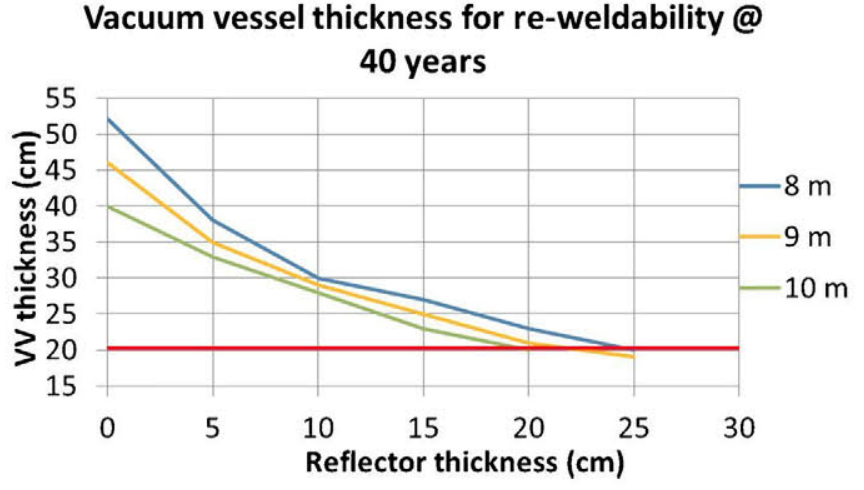

Fig. 4. Vacuum Vessel thickness as a function of the neutron reflector thickness required for reweldability of the external surface after 40 years of maintained irradiation. It is computed for different VV inner radius. This study is done for the thin blanket reaction chamber.

\section{Conclusions}

\subsection{Thin blanket vs. thick blanket}

We emphasize here the advantages of each blanket option. The thin blanket makes use of a significantly shorter volume of $\mathrm{LiPb}, 310 \mathrm{~m}^{3}$ versus $475 \mathrm{~m}^{3}$. This has obvious structural advantages, although it still requires a structural analysis to determine its structural viability. In addition, the radiological threatening inventory ( $\mathrm{T}$, ${ }^{210} \mathrm{Po},{ }^{203} \mathrm{Hg}$ ) is lower in the case of the thin blanket, relaxing the confinement requirements of safety systems.

On the other hand, the thick blanket operates at lower ${ }^{6} \mathrm{Li}$ enrichment, what means a cheaper operation of the facility. And the larger LiPb volume offers increased radiological protection for the VV against neutrons.

However, the VV thickness is a common problem for both options, as a thickness larger than $20 \mathrm{~cm}$ is to be avoided. Consequently, this advantage of the thick blanket is insufficient to compensate the worse performance in term of radiological inventories. The DRC can be reduced by means of online extraction systems [3], and the waste stream will be smaller in the case of the thin blanket. The thin blanket is selected for further design evolution, remarking the fact that the VV will require a specific attention.

\subsection{Vacuum Vessel}

In order to better protect the $\mathrm{VV}$ from the neutron irradiation and to keep the VV thickness below $20 \mathrm{~cm}$, the impact of the VV distance to the ignitions and a graphite neutron reflector was studied.

The effect of increasing the VV inner radius is larger when the neutron graphite reflector is smaller. However, in order to attain a VV thickness below $20 \mathrm{~cm}$ for the thin blanket, a reflector thickness between 20 and $25 \mathrm{~cm}$ is required for $\mathrm{VV}$ inner radius from 8 to $10 \mathrm{~m}$.

\section{Acknowledgments}

This work is funded by the Spanish "Programa de internacionalización de la $\mathrm{I}+\mathrm{D}$, Subprograma relativo a infraestructuras científicas internacionales", AIC-A-2011-0726, of Ministerio de Economía y Competitividad.

\section{References}

[1] R. Juarez, J. Sanz, J.M. Perlado, B. Le-Garrec, Fusion Engineering and Design 86 (2011) 694-698.

[2] L. El-Guebaly, S. Malang, Fusion Engineering and Design 84 (2009) 2072-2083. 
[3] D.A. Petti, B.J. Merrill, R.L. Moore, G.R. Longhurst, L. El-Guebaly, E. Mogahed, et al., Fusion Engineering and Design 80 (2006) 111-137.

[4] DOE STD 6003-96, The Safety of Magnetic Fusion Facilities, 1996.

[5] J.F. Latkowski, R.P. Abbott, S. Aceves, T. Anklam, A.W. Cook, ]. DeMuth, et al., Fusion Science and Technology 60 (2011) 565-569.

[6] M.E. Sawan, I.N. Sviatoslavsky, A.R. Raffray, X. Wang, Neutronic assessment of blanket options for the HAPI. laser inertial fusion energy chamber, in: Contribution to the twenty-first IEEE/NPS Symposium on Fusion Engineering. IEEE Xplore, 2005.

[7] J.Alvarez, A. Rivera, R. Gonzalez-Arrabal, D. Garoz, E. del Rio, ].M. Perlado, Fusion Science and Technology 60 (2011) 565-569

[8] R. Juárez, J. Sanz, J. Hernández, C.Sánchez, C.Zanzi, J.M. Perlado, Studies of a self cooled lead lithium blanket for HiPER reactor, in: Contribution to IFSA, 2011.

[9] D. Maisonnier, I. Cook, P. Sardain, L. Boccaccini, E. Bogusch, K. Broden, et al., Fusion Engineering and Design 75-79 (2005) 1173-1179.
[10] Y. Wu, F.D.S Team, Fusion Engineering and Design 84 (2009) 19871992.

[11] D.B. Pelowitz, MCNPX User's Manual, LA-CP-05-0369 (2005) and extensions.

[12] J. Sanz, O. Cabellos, N. Garcia-Herranz, ACAB-2008: Inventory code for nuclear applications., NEA-1839.

[13] A. Santamarina, D. Bernard, P. Blaise, M. Coste, A. Courcelle, T.D. Huynh, et al., NEA No 6807, ISBN 978-92-64-99074-6.

[14] J.-Ch. Sublet, L.W. Packer, J. Kopecky, R.A. Forrest, A.J. Koning, D.A. Rochman, culham Science Centre, Rept. CCFE-R(10) 05.

[15] O. Cabellos, ENDF/B-VII.0 data processed with NJOY-99.259 for radiation dam age calculations with extensions to important reactions for activation, NEA Data Bank, 2007

[16] R. Lindau, A. Möslang, M. Rieth, M. Klimiankou, E. Materna-Morris, A. Alamo, t al., Fusion Engineering and Design 75-79 (2005) 989-996.

[17] http://aries.ucsd.edu/ARIES/WDOCS/ARIES-IFE/SPECTRA/. 